\section{Resistance to consistent shocks as a function of training schedules of interspersed shocks ${ }^{1}$}

\author{
M. AKHTAR ${ }^{2}$ and T. AHSAN, University \\ of Karachi, Karachi 32, Pakistan
}

An earlier study had developed a response that persisted in spite of shocks-no-food for 2 months. The present study successfully replicated the inextinguishable character of the response (Experiment 1) and, as one of its determinants, further investigated three schedules of shocks interspersed among rewards: (1) fixed ratio - constant frequency, (2) random ratio - constant frequency, and (3) gradual progression. Tests with consistent shocks showed: (1) no differences between fixed and random ratio and (2) a greater suppression in gradual progression, suggesting schedule as subsidiary to frequency of shocks.

A previous study (Akhtar, 1967) had attained an extremely heightened resistance to consistent shocks unaccompanied by food reward. Rats that were trained with shocks interspersed among rewards for 1 week continued lever-pressing until the end of a test that lasted for nearly 2 months. This finding, paradoxical in a sense, is of particular significance, notwithstanding earlier findings (Muenzinger, 1934; Farber, 1948; Maier, 1949) of similarly paradoxical phenomena shown in visual discrimination situations (see review by Church, 1963) or more recent findings by Holtz \& Azrin (1961). The study by Holtz and Azrin, quite cleverly designed in its own way, strikes one as somewhat less dramatic in one sense, as 30-min punishment sessions were carried out in the presence of reward schedules and were alternated by $120-\mathrm{min}$ sessions of no punishment-no food. The discrimination situation of a T-maze-used in earlier studies-is generally more complex than a simple operant (Mowrer, 1960; Spence, 1960). The experimental situation used by Maier to study frustration is still more complicated and presupposes an understanding of many stimulus parameters that is not yet available (Dinsmoor, 1960). Reviewing findings on frustration, Yates (1962) emphasized the need of understanding the effects of various schedules of punishment as one such a parameter.

In view of its considerable importance, the finding of a persistent performance obtained with a simple operant (Akhtar, 1967) needed to be replicated. After settling the problem of replicability, the present study aimed to isolate the effects of two schedules of interspersing shocks. The need for a study of two schedules arose also from one other source, the rationale for which is presented in the following section.

The condition in the previous study (Akhtar, 1967), designated as IPf, that resulted in a response persisting in the face of consistent punishments for 2 months, consisted of interspersed punishments given in a fixed schedule; every fourth response was shocked, in contrast to the condition of a variable schedule, designated as IP-7, in which interspersed punishments started with two shocks on the first day and increased in a gradual progression to nine shocks on the last day in the daily total of 20 trials. The IP-7 group reached the criterion of suppression in about a week when tested with consistent punishments. The two groups received training for 7 days with an equal total of interspersed shocks. But the frequency of shocks interspersed per day was unequal in the two groups. The present study attempts to control this factor so that effects due to schedules can be isolated unambiguously.

\section{EXPERIMENTAL DESIGN}

There were two groups: IPf, exactly like the fixed schedule in the previous study, and IPr (random schedule). Both groups received five shocks per day; for $\mathrm{IPf}$, a shocked trial was fixed (every fourth), while for IPr, it was determined randomly.

SUBJECTS AND APPARATUS

Seven rats were assigned randomly to each of the two groups. They were all male Holtzman rats, approximately 90 days old at the beginning of the experiment. Within the close range of $320-342 \mathrm{~g}$, the mean weight for IPf was $331 \mathrm{~g}$ and for IPr $333 \mathrm{~g}$.

A Skinner box was modified, and its grid floor was electrified by a current of nearly $.23 \mathrm{~mA}$. Foot shock lasted $.25 \mathrm{sec}$. PROCEDURE

Adaptation (10 Days)

The Ss were handled for 2 days and were adapted to a deprivation schedule that maintained their weights at $80 \%$ of the initial body weights by feeding them a necessary amount of wet, mashed Purina chow that varied around the mean of $11 \mathrm{~g}$.

Approximation Training (6 Days)

By the end of 6 days, Ss had leamed to bar-press by themselves. Mean rate of pressing for IPf was 8.0, which did not differ from the mean rate of 8.4 for IPr or the mean rate of 7.0 for IPf in the previous study (Akhtar, 1967).
Acquisition Training (7 Days)

A daily series of 20 presses was allowed. IPf received shocks on every fourth response, followed by three rewarded trials. IPr received five shocks on the trials that were determined randomly for each day and were as follows: Day $1-3,7,13$, 14, 16; Day 2-3, 5, 10, 12, 14; Day 3-6, $16,17,18,20$; Day 4-4, 6, 16, 17, 19; Day 5-7, 10, 12, 13, 15; Day 6-7, 9, 16, 17,18 ; Day $7-3,6,7,10,16$.

lest with Consistent Shocks (17 Days)

Every trial was shocked in absence of food reward. Twenty trials were allowed daily. On Day $11 / 14$, Ss, after finishing 20 shocked presses, were detained for $1 / 2 \mathrm{~h}$ before feeding. On Day 15/17, like earlier, they were fed immediately the daily ration of food in their home cages.

RESULTS AND DISCUSSION

Rates of bar pressing during acquisition and test were analyzed by the Mann-Whitney U test. Performance of IPf will be reported first in comparison to the performance of IPr and next in comparison to the performance of IPf of the previous study.

\section{IPf vs IPr}

Acquisition training. There were no differences in the two groups on any of the 7 days. Mean rate in IPf was 11.2 on Day 1 and 14.0 on Day 7; probabilities of differences were, respectively, .45 and .50 . IPf showed a tendency to be slowest on Day 2. Both groups gradually recovered from the suppressive effects of shocks as days of training progressed.

Test with consistent shocks. Test performance of two groups is shown in Fig. 1. The two groups did not differ from each other. Probability of their differences was around .3 and .4 for most of the days.

Detention, rather than an immediate feeding, did not show any effect. Comparing performance for Days 15/17 (postdetention) with one's own performance for Days 11/14 (detention) did not reveal any difference; in the postdetention period, six Ss were faster and eight $S s$ were slower in both the groups combined. Instead, performance during detention days $(11 / 14)$ as compared to predetention days (8-10) was faster in IPf with a $p<.07$ and in IPI with a $p<.06$, and in both the groups combined with a $\mathrm{p}<.01$. This indicated an improving tendency with a continued test (also found in Akhtar, 1967), on the one hand, and an ineffectiveness of the detention treatment, on the other hand. This lack of effect of detention in the present study would appear to be due to the fact that detention was introduced early in the test (i.e., after 10 days), by which time expectancy of immediate feeding was not very strong. 


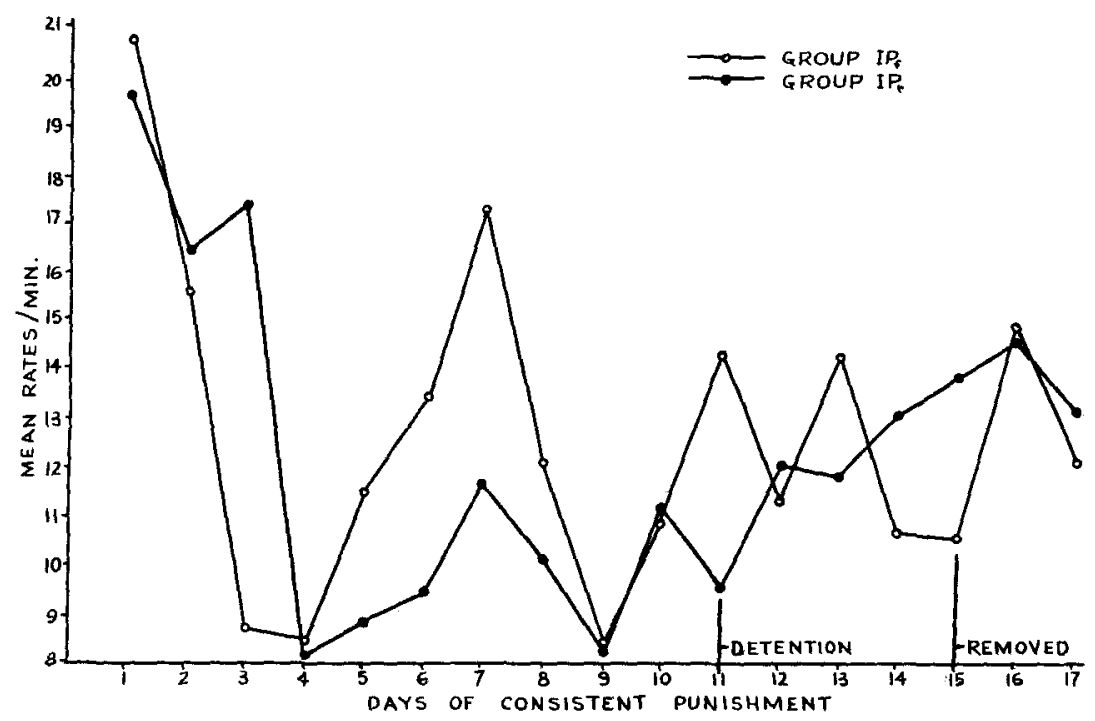

This assumption was made in Akhtar \& Bond (1968) in keeping with their finding of a significant retardation in the test performance when detention for 5 days was introduced after 39 days of immediate feeding given after the test performance.

IPf in the present vs previous study. The rats in the present study were somewhat more agile in general and were consistently faster than previously in all the phases: At the end of preliminary training, $p$ value of the differences was .2 (two-tailed test); for most of the acquisition days, $p$ was around .3 , while it was $<.05$ and $<.18$, respectively, for Day 4 and Day 5 of acquisition; during testing, $p$ reached significance $(.05)$ for Days $3,5,7$, and 17 . In the present sample, all seven Ss (100\%) were performing at a high level by Day 17 in comparison to the previous sample, in which 2 out of $12 \mathrm{Ss}$ had reached the criterion of cessation (zero response) by this time and only $83 \%$ were performing, and continued to improve with the progress of testing. Hence, it was sufficiently indicated that a $S$ that did not reach suppression by Day 17 of the test should be expected to continue indefinitely, e.g., by Day 48 , as testing was carried out in the previous study. In the previous sample, one $S$ reached the criterion on Day 7 and the other $S$ reached it on Day 16, and both the Ss had exhibited very low rates from an early period of testing. There was no such indication of a tendency to suppression in the present sample. A tendency to improve in test performance appeared to set in after Day 10 in both the samples-provided a $S$ had not developed suppression by this time. The fact that the present sample showed a significantly higher performance during the test should be taken as additional assurance that the present sample would be at least as inextinguishable as the previous one. It can be safely concluded, then, within reasonable limits, that the present sample replicates successfully an inextinguishable response similar to that shown in the previous sample.

\section{EXPERIMENT 2}

The equal performance of IPf and IPr shown in Experiment 1 disagrees with the explanation offered in the previous study for the heightened resistance of IPf in comparison to that of IP-7 (Akhtar, 1967). The explanation given there attributed the differences to the fixed schedule of interspersing shocks, which, by increasing predictability of shocks, was assumed to have enhanced resistance in IPf in comparison to the variable schedule in IP-7. This would suggest a different interpretation that emphasizes the basis for differential resistance in IPf and IP-7 more in the characteristic schedule of IP-7, namely, the fact that in a gradual progression, IP-7 received a higher frequency of shocks for Days $5 / 7$ of the training. In order to strengthen the proposed interpretation, the role of inequality in frequency of shocks per day was directly investigated in two schedules in Experiment 2, in which the variable mode of shocking was held constant. Two conditions, gradual-variable (GV) and random-constant (RC), were used that, respectively, were like IP-7 of the previous study and IPr of Experiment 1. Both groups received shocks on unpredictable trials; frequency of shocks for RC was the same each day, while it increased gradually for GV over successive days of training. A study of gradual increase in shocks is of an independent interest in view of its assumed high adaptability value--a notion that is
Fig. 1. An equal test performance in the two groups.

deeply ingrained in experimental psychology.

SUBJECTS AND APPARATUS

Sixteen male albino rats from the Sprague-Dawley strain, bred in the colony of the University of Karachi, were assigned randomly to the two groups. The intensity of shock was $.35 \mathrm{~mA}$, and its duration was $.25 \mathrm{sec}$.

All other details of procedure, approximation training (6 days), acquisition training ( 7 days), and the trial numbers that were shocked for $\mathrm{RC}$, were the same as given in Experiment 1. The RC group received five shocks per day-a frequency equal to the mean number of shocks received by GV. Shocks in GV, however, increased gradually from one shock the 1st day to eight shocks the 7 th day of training. The exact schedule of shocking for GV was as follows: Day 1-1:10 (to be read as one shock on Trial 10); Day 2-2:7, 13 (two shocks given on Trials 7 and 13); Day 3-4:4, 7, 12, 18; Day 4-5:3, 6, 10, 14, 18; Day 5-6:2, 6, 7, $11,15,17$; Day 6-7:3, 5, 6, 10, 15, 16, 17; Day 7-8:2, 4, 7, 8, 9, 13, 15, 18. After 7 days of training, both groups were tested for 10 days. During the test, each press resulted in a shock and no food. S was allowed 20 presses or $10 \mathrm{~min}$, whichever came first.

\section{RESULTS}

Acquisition Training

The RC group received five shocks constantly and was therefore slower than GV Day $1(\mathrm{p}<.01)$ and Day $2(\mathrm{p}<.02)$, when the latter group received one and two shocks, respectively. Differences were insignificant any other day but were systematic in accordance with frequency of shocks: They favored GV for Day 3 and Day 4, after which the number of shocks received by $\mathrm{GV}$ increased, making it slower than RC.

\section{Testing}

Mean rates of presses for both $\mathrm{RC}$ and GV are shown in Fig. 2. Rates of presses of the two groups showed a significant heterogeneity of variance and were therefore analyzed by the Kolmogorov-Smirnov test. Differences reached significance $(p<.05)$ for each of the first 3 days or for all days combined.

Performance of IPr from Experiment 1 and of RC from Experiment 2 can be directly compared for the difference in intensity of shock- $.23 \mathrm{~mA}$ and $.35 \mathrm{~mA}$, respectively; every other variable was constant. Test performance with lower intensity (in Experiment 1) was faster than that with higher intensity: significantly for Days $1-2$, insignificantly for Days 3-5, and 


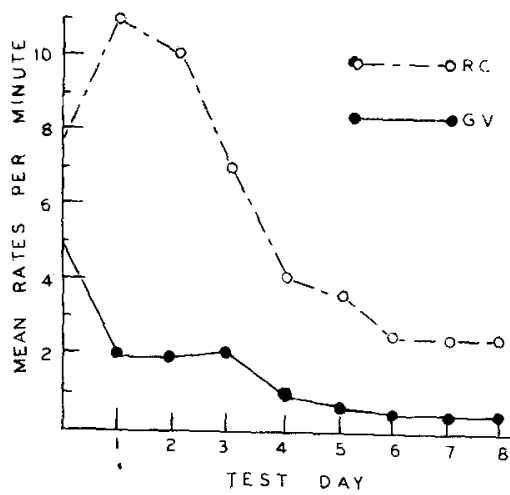

Fig. 2. A lower performance in the gradual group.

again significantly for Days 6-10. By the end of test, all (100\%) Ss from IPr were responding, in contrast to only $50 \%$ Ss from RC.

\section{DISCUSSION}

It was evident that a gradual progression of interspersed shocks decreased rather than increased resistance to consistent shocks. This further confirmed the interpretation from Experiment 1 that the basis for a superior resistance in IPf to that in IP.7 (Akhtar, 1967) was in the gradual schedule of IP-7 and not in the fixed schedule of IPf. Hardly any other study of frequency of interspersed shocks has directly evaluated the effects of gradual vs constant schedule. Miller (1960) and Ferstein \& Miller (1963) have shown that the gradual group was more resistant than the sudden group. But these groups actually presented contrast between a prior experience and no experience with shocks-a variable that should be held constant to enable a comparison strictly on the variable of gradualness.

Even though no other direct evidence on a gradual increase in frequency of shocks is available, the present finding of a reduced resistance may seem surprising, as it may appear to run contrary to the notion of adaptation that is widely held both by theorists and experimentalists. "Punishment intensity if slowly increased," theorizes Solomon, "tends not to be as effective as in the case where it is introduced initially at its high intensity value [1964, p. 241]." The factor of gradualness has been similarly emphasized also by Martin (1963, p. 451). It has been a popular practice among experimentalists to increase intensity in a gradual progression
(Miller, 1960; Logan, 1960; Bower \& Miller, 1960; Brown \& Wagner, 1964; Kintz et al, 1967) in an attempt, implicit in some, to minimize the suppressive effects of shocks. This practice is probably a valid strategy of keeping aversive effects as minimal, as indeed has been demonstrated by Melvin \& Bender (1968), provided that the gradual group finally stops at an intensity that the constant group had experienced all along. This, however, would leave an inequality in the two groups in the total amount of intensity. Having experienced a lesser intensity during the early phases of training, the gradual group would be expected to be less fearful simply on account of an intensity variable (Karsch, 1962). Now, if the mean or total intensity experienced is to be equated, intensity will start initially at a lower level in the gradual group but will inevitably have to end up at a level higher than that in the constant group--analogous to a higher frequency of shocks in GV-and would thus lead to predict a greater suppression. To clarify, both the conflicting predictions can, perhaps, be reconciled on the basis of a crucial variable of intensity (or frequency) of shocks, which will work in one way if only the final level of shocks is equated and in another way if the mean level of shocks is equated.

Finally, relevance of the present findings may be pointed out for Logan \& Wagner's theory (1965, pp. 54-56). Logan et al (1955) found that rats ran more slowly when the reward was nine pellets half the time and one pellet half the time, in comparison to a constant five-pellet reward. They suggested a rule that the incentive value of a varied reward or punishment is less than that produced by a constant reward or punishment. The present findings showed clearly that the variable-gradual shocks were more suppressive (i.e., had a higher incentive value) than were an equal mean frequency of shocks given constantly.

\section{REFERENCES}

AKHTAR, M. Increased resistance to punishment as a function of counter conditioning. Journal of Comparative \& Physiological Psychology, $1967,64,268-272$

AKHTAR, M., \& BOND, E. The role of anticipation of post-session feeding in increasing resistance to punishment. Psychonomic Science, 1968, 12, 347-348.

BOWER, G. H., \& MILLER, N. E. Effects of amount of reward on strength of approach in an approach-avoidance conflict. Journal of Comparative \& Physiological Psychology, $1960,53,59-62$.

BROWN, R. I., \& WAGNER, A. R. Resistance to punishment and extinction following training with shock and/or non-reinforcement. Journal of Experimental Psychology, 1964, 68, 503-507.

CHURCH, R. M. The varied effects of punishment on behavior. Psychological Review, 1963, 70, 369-402.

DINSMOOR, J. A. Abnormal behavior in animals. In R. Waters et al (Eds.), Principles of comparative psychology. New York: McGraw-Hill, 1960. Pp. 289-324.

FARBER, I. E. Response fixation under anxiety and nonanxiety condition. Journal or Experimental Psychology, 1948, 38, 111-131. FERSTEIN, A., \& MILLER, N. E. Learning to resist pain and fear: Shock before versus after reaching goal. Journal of Comparative \& Physiological Psychology, 1963, 56, 797.

HOLTZ, W. C., \& AZRIN, N. H. Discriminative properties of punishment. Journal of the Experimental Analysis of Behavior, 1961, 4, 225-232.

KARSH, E. Effects of number of rewarded trials and intensity of punishment on running speed. Journal of Comparative \& Physiological Psychology, 1962, 55, 44.51.

KINTZ, B. L., et al. Punishment and compulsive avoidance behavior. Journal of Comparative \& Physiological Psychology, 1967, 63, 323-326.

LOGAN, F. A., et al. Effects of varied reinforcement on speed of locomotion. Journal of Experimental Psychology, 1955, $49,260-266$

LOGAN, F. A. Incentive. New Haven: Yale University Press, 1960.

LOGAN, F. A., \& WAGNER, A. R. Reward and punishment. Boston: Allyn \& Bacon, 1965.

MAIER, N. R. F. Frustration: The study of behavior without a goal. New York: McGraw-Hill, 1949.

MARTIN, B. Reward and punishment associated with the same goal response. Psychological Bulletin, 1963, 60, 441-451.

MELVIN, K., \& BENDER, L. Effects of changes in punishment intensity. Psychological Record, $1968,18,29-34$.

MILLER, N. E. Learning resistance to pain and fear. Journal of Experimental Psychology, $1960,60,137-145$.

MOWRER, O. H. Learning theory and behavior. New York: Wiley, 1960 .

MUENZINGER, K. F. Motivation in learning. I. Journal of Comparative \& Physiological Psychology, 1934, 17, 267-277.

SOLOMON, R. L. Punishment. American Psychologist, 1964, 19, 239-253.

SPENCE, K. W. Behavior theory and learning. Englewood Cliffs, N.J: Prentice-Hall, 1960.

YATES, A. Frustration and conflict. New York: Wiley, 1962.

\section{NOTES}

1. A portion of the present research was presented at the meetings of the 19th International Congress of Psychology held in London, July 1969.

2. Requests for reprints should be sent to $M$. Akhtar, Department of Psychology, State College at Slippery Rock, Pennsylvania 16057. 